\title{
An Open Letter Arguing for Closure of the Practice Pathway for Clinical Informatics Medical Subspecialty Certification
}

\author{
Robert W. Turer ${ }^{1}$ Bruce P. Levy ${ }^{2}$ Jonathan D. Hron ${ }^{3}$ \\ Christoph U. Lehmann ${ }^{1}$ \\ ${ }^{1}$ Clinical Informatics Center, UTSouthwestern Medical Center, Dallas, \\ Texas, United States \\ 2 Division of Clinical Informatics, Department of Pathology, Geisinger \\ Medical Center, Danville, Pennsylvania, United States \\ ${ }^{3}$ Department of Pediatrics, Boston Children's Hospital, Boston, \\ Massachusetts, United States \\ ${ }^{4}$ Division of Pediatric Critical Care, Department of Pediatrics, \\ Stanford Children's Health, Palo Alto, California, United States \\ ${ }^{5}$ Department of Biomedical Informatics, Vanderbilt University \\ Medical Center, Nashville, Tennessee, United States \\ 6 Department of Pathology, Mass General Brigham, Boston, \\ Massachusetts, United States \\ 7 Department of Radiation Oncology, Brigham and Women's Hospital, \\ Boston, Massachusetts, United States
}

Appl Clin Inform 2022;13:301-303.

We would like to thank the Members of the American Board of Preventive Medicine (ABPM) and the American Board of Pathology (ABPath) for their past contributions to the medical subspecialty of clinical informatics $(\mathrm{CI})$ and their continued interest in the specialty's ongoing growth and success. We recently learned that the Boards are considering an extension of the Practice Pathway for $\mathrm{Cl}$ certification beyond 2022. We write this open letter requesting that the Boards do not pursue this course of action.

For unfamiliar readers, CI leaders from the American Medical Informatics Association (AMIA) and other organizations collaborated with ABPM and ABPath to outline the requirements for $\mathrm{CI}$ medical subspecialty certification in the early 2000s. ${ }^{1-4}$ The formal subspecialty was born with the successful certification of the first class of diplomates in January 2014. ${ }^{5}$ Since that first class, approximately 2,000 physicians have successfully completed certification, with the majority becoming certified through the Practice Pathway instead Fellowship Pathway. ${ }^{6,7}$ During the early years of the specialty, there was a clear need for practicing informaticians to become certified without retraining, as had been the case with other new specialties, such as emergency medicine. For other specialties, however, those practice pathways closed within roughly a decade with the residual
Address for correspondence Robert W. Turer, MD, Department of Emergency Medicine and Clinical Informatics Center, UT Southwestern Medical Center, 5323 Harry Hines Boulevard, Suite E4.300, Dallas, TX 75390, United States (e-mail: robert.turer@utsouthwestern.edu).

expectation being to complete formal residency or fellowship training prior to board eligibility. ${ }^{8-10}$

The ABPM CI Practice Pathway requires informatics practice for at least $25 \%$ of a full-time equivalent (FTE) for 3 years or either a Masters or PhD in biomedical informatics. ${ }^{11}$ The practice pathway's $25 \%$ FTE equivalent is not as rigorous as prior specialty boards' practice pathways, such as the American Board of Emergency Medicine's, which required 7,000 hours practicing and teaching emergency medicine over a minimum of 5 years with 2,800 hours within any 24-month period prior to application, as well as 50 hours of approved continuing medical education annually since 1973 when their application opened. ${ }^{10}$ While masters programs might provide a reasonable knowledge-based foundation for a career in $\mathrm{CI}$, they do not provide comparable applied experience to fellowship programs. As an analogy, while some clinicians with clinical training might be able to study for 6 months and pass a board examination in critical care or cardiology, doing so would not prepare them for the safe practice of either specialty without the practical and supervised experience obtained during fellowship training. It is that experiential learning, not just book and examinationbased learning, which prepares physicians for the safe practice of medicine in any specialty including Cl. Effectiveness of received

February 18, 2022

accepted

February 18, 2022 (c) 2022. Thieme. All rights reserved. Georg Thieme Verlag KG,

Rüdigerstraße 14,

70469 Stuttgart, Germany
DOI https://doi.org/ $10.1055 / \mathrm{s}-0042-1744386$. ISSN 1869-0327. 
fellowship training has also been corroborated by higher pass rates for fellowship-trained applicants compared with those seeking certification through the Practice Pathway. For instance, an internal communication on the AMIA Community of Clinical Informatics Program Directors (CIPD) message board reported that the pass rate among the 38 fellowshiptrained examinees who took the examination in 2020 was $95 \%$, while the overall pass rate of all 273 examinees (including the 38 fellowship-trained applicants) was $84 \%$. By proxy, this means that the pass rate among the 235 Practice Pathway examinees was $82 \%$.

AMIA's web site states, "Becoming board-certified in clinical informatics sets the highest bar for those who are experts in the subspecialty. It provides the opportunity to demonstrate your knowledge applying informatics to deliver improved healthcare services." 12 Given the two decades of effort writing and refining the subspecialty's delineation of practice ${ }^{4,13,14}$ and training expectations embodied in the Accreditation Council for Graduate Medical Education (ACGME) Program Requirements, ${ }^{15}$ we have truly set a high bar for our fellowship graduates and built the infrastructure to train our future leaders. If we expect this level of skill and rigor from our colleagues, we should require new diplomates to be fellowship trained as is the case with every other medical specialty. The practice pathway was intended for already practicing physicians at the initiation of a new board, not as an alternate pathway for physicians who are unwilling to pursue a fellowship more than a decade later. Experienced clinical informaticians have had ample time to take the certification examination. We hope moving forward that the Boards will share our opinion that quality is more important than quantity with respect to board certification.

While it might make sense to have concerns about closing the practice pathway if fellowship programs could not meet the demand for trainees, the opposite is in fact the case. The number of ACGME-accredited CI fellowships has grown from four accredited programs to an astonishing 57 in less than 8 years, despite many launching during a global pandemic. ${ }^{16}$ For context, this number is equivalent to or greater than most other preventive medicine or pathology specialties which have existed for decades. ${ }^{16}$ In the context of this success, the extended practice pathway hinders $\mathrm{CI}$ fellowships' abilities to recruit the best candidates, as candidates opt instead to pursue less rigorous, less costly, and more convenient training options. However, inferior training is damaging for the specialty in the end, and the continued reliance on educational programs with minimal, often unsupervised practical experience dilutes the value of our board certification and threatens the viability of fellowship programs across the country.

Another argument potentially used against closing the practice pathway might be that the focused, concentrated nature of CI fellowships could discourage members of surgical or other subspecialties from pursuing fellowship training. However, in partnership with the residency and fellowship programs, ABPM and ABPath have creatively addressed many of these concerns by accommodating integrated training pathways ${ }^{17}$ and allowing surgical residents to complete $\mathrm{CI}$ training during research years despite not being boarded in a primary specialty. ${ }^{18}$ These solutions make our subspecialty inclusive without diluting the rigor of our fellowships or the value of board certification, and we applaud the Boards' efforts in this regard.

Beyond the merit-based reasons for not extending the Practice Pathway is a concern of trust. To those of us who trusted ABPM and ABPath when choosing to pursue $\mathrm{CI}$ fellowships or to start fellowship programs after being assured that fellowship training would become the expected standard for our specialty (as documented in writing now for almost a decade), it would constitute a tremendous breach of trust if the rules changed now.

The long-term harms of extending the Practice Pathway for CI board eligibility beyond 2022 far outweigh any shortterm gains. We thank ABPM and ABPath for all they have done for the $\mathrm{Cl}$ subspecialty, and we recommend that the Practice Pathway not be extended. We hope the Boards will make the right decision.

Protection of Human and Animal Subjects

No human subject research was performed for this letter.

Conflict of Interest

E.K. reports grants or contracts from any entity National Library of Medicine, National Institutes of Health-Building Interdisciplinary Research Team (BIRT) Award, grant number: 45614187; leadership or fiduciary role in other board, society, committee or advocacy group, paid, or unpaid from Chair of Physicians in the American Medical Informatics Association (AMIA) Community. B.P.L. reports leadership or fiduciary role in other board, society, committee or advocacy group, paid, or unpaid from Chair of Physicians in AMIA Community. C.U.L. reports royalties or licenses from Springer-Verlag $(<\$ 20)$; leadership or fiduciary role in other board, society, committee or advocacy group, paid, or unpaid from Past President International Medical Informatics Association; and Stock or stock options from Celanese, Markel.

\section{Acknowledgments}

We thank Dr. Michael Leu, Chair of the American Medical Informatics Association's (AMIA's) Community of Informatics Program Directors (CIPD), for his careful review of this letter.

\section{References}

1 Detmer DE, Munger BS, Lehmann CU. Clinical informatics board certification: history, current status, and predicted impact on the clinical informatics workforce. Appl Clin Inform 2010;1(01): $11-18$

2 Lehmann CU, Silverman HD, Gardner RM, Safran C, Gadd C. Clinical Informatics Subspecialty Certification and Training. In: Berner ES, ed. Informatics Education in Healthcare. 2nd ed. Switzerland: Springer; 2020:213-235

3 Gardner RM, Overhage JM, Steen EB, et al; AMIA Board of Directors. Core content for the subspecialty of clinical informatics. J Am Med Inform Assoc 2009;16(02):153-157

4 Safran C, Shabot MM, Munger BS, et al; AMIA Board of Directors. Program requirements for fellowship education in the 
subspecialty of clinical informatics. J Am Med Inform Assoc 2009; 16(02):158-166

5 Middleton B. First diplomates board certified in the subspecialty of clinical informatics. J Am Med Inform Assoc 2014;21(02):384

6 Lehmann CU, Gundlapalli AV, Williamson JJ, et al. Five years of clinical informatics board certification for physicians in the United States of America. Yearb Med Inform 2018;27(01):237-242

7 Desai S, Mostaghimi A, Nambudiri VE. Clinical informatics subspecialists: characterizing a novel evolving workforce. J Am Med Inform Assoc 2020;27(11):1711-1715

8 American Board of Emergency Medicine. ABEM History. Accessed February 16, 2022 at: https://www.abem.org/public/about-abe$\mathrm{m} /$ abem-history

9 Weiss LD. Common Sense: The Newsletter of the American Academy of Emergency Medicine. Accessed February 22, 2022 at: https://www.aaem.org/UserFiles/file/commonsense0110.pdf

10 Daniel v. Am. Bd. of Emergency Med. , 428 F.3d 428: United States Court of Appeals, Second Circuit 2005

11 American Board of Preventative Medicine. Clinical informatics. https://www.theabpm.org/become-certified/subspecialties/clinical-informatics/. Accessed February 16, 2022

12 American Medical Informatics Association. Clinical informatics subspecialty. Accessed February 16, 2022 at: https://amia.org/careers-certifications/clinical-informatics-subspecialty

13 Silverman HD, Steen EB, Carpenito JN, Ondrula CJ, Williamson JJ, Fridsma DB. Domains, tasks, and knowledge for clinical informat- ics subspecialty practice: results of a practice analysis. J Am Med Inform Assoc 2019;26(07):586-593

14 Detmer DE, Lumpkin JR, Williamson JJ. Defining the medical subspecialty of clinical informatics. J Am Med Inform Assoc 2009;16(02):167-168

15 ACGME International. Advanced specialty program requirements for graduate medical education in clinical informatics. Accessed February 16, 2022 at: https://www.acgme-i.org/Portals/0/Specialties/Clinical\%20Informatics/Clinical\%20Informatics.pdf? ver=2018-06-11-102709-603

16 Accredidation Council for Graduate Medical Education. Institution and Program Finder. Accessed February 16, 2022 at: https:// apps.acgme.org/ads/public/

17 Palma JP, Hron JD, Luberti AA. Early experiences with combined fellowship training in clinical informatics. J Am Med Inform Assoc 2020;27(05):788-792

18 American Board of Preventative Medicine. The American Board of Preventive Medicine has obtained approval from the American Board of Medical Specialties' (ABMS) to offer surgical residents Mid-Residency Training Programs in Clinical Informatics (MRTP). Accessed February 16, 2022 at: https://www.theabpm.org/ 2019/03/19/the-american-board-of-preventive-medicine-hasobtained-approval-from-the-american-board-of-medical-specialties-abms-to-offer-surgical-residents-mid-residency-training-programs-in-clinical-informat/ 